Algebraic $8 \mathcal{G}$ Geometric $\mathcal{T}$ opology

Volume 4 (2004) 571-594

Published: 8 August 2004

ATG

\title{
Links associated with generic immersions of graphs
}

\author{
TOMOMI KaWAMURA
}

\begin{abstract}
As an extension of the class of algebraic links, A'Campo, Gibson, and Ishikawa constructed links associated to immersed arcs and trees in a two-dimensional disk. By extending their arguments, we construct links associated to immersed graphs in a disk, and show that such links are quasipositive.
\end{abstract}

AMS Classification 57M25; 57M27

Keywords Divide, graph divide, quasipositive link, slice Euler characteristic, four-dimensional clasp number

\section{Introduction}

In 11, A'Campo constructed links of divides as an extension of the class of algebraic links. A divide is a generic relative immersion of a disjoint union of arcs (and loops) in a 2-dimensional disk. In [7, Gibson and Ishikawa constructed links of free divides, non-relative immersions of intervals in a 2-dimensional disk. We review links of divides in Section 2] and remark on links of free divides in Section 3 ,

In 2, A'Campo showed that any divide link is ambient isotopic to a transverse $\mathbb{C}$-link. A transverse $\mathbb{C}$-link is the link represented as the transversal intersection of an algebraic curve and the unit sphere in the 2-dimensional complex space $\mathbb{C}^{2}$ [15]. An algebraic link is the link of a singularity of an algebraic curve. An algebraic link is a transverse $\mathbb{C}$-link; there exist transverse $\mathbb{C}$-links which are not algebraic [14].

In [12, the author showed that links of divides and free divides are quasipositive, by using the visualization algorithm due to Hirasawa [8]. A quasipositive braid is a product of braids which are conjugates of positive braids, and a quasipositive link is an oriented link which has a closed quasipositive braid diagram. A positive braid is a product of canonical generators of the braid group, that is, a braid which has a diagram without negative crossings. It is well known that any algebraic link admits a representation as a closed positive braid. In 14, 
Rudolph showed that quasipositive links are transverse $\mathbb{C}$-links. In [4, Boileau and Orevkov proved that transverse $\mathbb{C}$-links are quasipositive, by using the theory of pseudoholomorphic curves.

There exist quasipositive links which are not the links of any divides or free divides [12. For classification of such links, we are interested in extending the class of links of divides and free divides. In [5], Gibson associated links with generic immersions of trees in a 2-dimensional disk. A tree divide is such an immersion of trees. He suggested to the author the quasipositivity problem for such links. In this paper, we construct graph divide links as an extension of the class of links of divides or free divides in Section 3, show that tree divide links constructed by Gibson can be represented as graph divide links in Section 4 and prove that such links are quasipositive in Section 5. Furthermore we determine some geometric invariants for graph divide links, and show that there exists a quasipositive link which is not a graph divide link.

Acknowledgment The author was partially supported by JSPS Research Fellowships for Young Scientists, while she was in Graduate School of Mathematical Sciences, University of Tokyo. She was also supported by Grant-in-Aid for Young Scientists (B) (No. 15740044), MEXT.

The author would like to thank Professor Masaharu Ishikawa and Doctor William Gibson for their helpful suggestions and useful comments about divides, free divides, tree divides, and graph divides. She also would like to thank them and Professor Toshitake Kohno for their encouragement. She would like to thank the referee for his/her pointing out the ambiguity in the first manuscript.

\section{Divide links and oriented divide links}

In this section, we review links of divides defined by A'Campo [1] and links of oriented divides defined by Gibson and Ishikawa [6].

By the argument due to A'Campo [1, a link is associated to any immersed arcs (and loops) in a disk as follows.

Let $D$ be a unit disk in the real plane $\mathbb{R}^{2}$, that is $D=\left\{x=\left(x_{1}, x_{2}\right) \in \mathbb{R}^{2} \mid\right.$ $\left.|x|^{2}=x_{1}^{2}+x_{2}^{2} \leq 1\right\}$. A divide $P$ is a generic relative immersion in the unit disk $(D, \partial D)$ of a finite number of 1 -manifolds, i.e., copies of the unit interval $(I, \partial I)$ and the unit circle [1, 2, 8, 10. We also call the image of such an immersion a divide. A branch of $P$ is each image of the copies. We shall call each image 
of the copies of the interval an interval branch, and each image of the copies of the circle a circle branch.

Let $T_{x} X$ be the tangent space at a point $x$ of a manifold $X$, and $T X$ be the tangent bundle over a manifold $X$. We identify the 3 -sphere $S^{3}$ with the set

$$
S T \mathbb{R}^{2}=\left\{\left.(x, u) \in T \mathbb{R}^{2}\left|x \in \mathbb{R}^{2}, u \in T_{x} \mathbb{R}^{2},\right| x\right|^{2}+|u|^{2}=1\right\} .
$$

The link of a divide $P$ is the set given by

$$
L(P)=\left\{(x, u) \in S T \mathbb{R}^{2} \mid x \in P, u \in T_{x} P\right\} .
$$

We orient the 3 -sphere and the link $L(P)$ as follows. We identify the tangent bundle $T \mathbb{R}^{2}=\mathbb{R}^{4}$ with the 2 -dimensional complex space $\mathbb{C}^{2}$ by the map

$$
\left(\left(x_{1}, x_{2}\right),\left(u_{1}, u_{2}\right)\right) \mapsto\left(x_{1}+\sqrt{-1} u_{1}, x_{2}+\sqrt{-1} u_{2}\right) .
$$

The tangent bundle $T \mathbb{R}^{2}$ is oriented by the complex orientation of $\mathbb{C}^{2}$, and the 3 -sphere is naturally oriented by the complex orientation of the 4 -ball

$$
\left\{\left.(x, u) \in T \mathbb{R}^{2}\left|x \in \mathbb{R}^{2}, u \in T_{x} \mathbb{R}^{2},\right| x\right|^{2}+|u|^{2} \leq 1\right\} .
$$

Let $[a, b]$ be a small interval with $a<b$. Let $\phi:[a, b] \rightarrow D$ be an embedding whose image lies on $P$. We orient a part of the link $L(P)$ as the image of the map $t \mapsto\left(\phi(t), \frac{\sqrt{1-|\phi(t)|^{2}}}{|\dot{\phi}(t)|} \dot{\phi}(t)\right)$, where $\dot{\phi}(t)$ is the differential of $\phi(t)$. We can extend this orientation to $L(P)$. A divide link is an oriented link ambient isotopic to the link of some divide.

In [6], Gibson and Ishikawa constructed links associated with oriented divides. An oriented divide is the image of a generic immersion of finite number of copies of the unit circle in the unit disk, with a specific orientation assigned to each immersed circle. The link $L_{\text {ori }}(Q)$ of an oriented divide $Q$ is defined by

$$
L_{\text {ori }}(Q)=\left\{(x, u) \in S T \mathbb{R}^{2} \mid x \in Q, u \in \vec{T}_{x} Q\right\},
$$

where $\vec{T}_{x} Q$ is the set of tangent vectors in the same direction as the assigned orientation of $Q$. The link $L_{\text {ori }}(Q)$ naturally inherits its orientation from $Q$. The ambient isotopy type of the link of an oriented divide does not change under the inverse self-tangency moves illustrated in Figure 1 (a) and (b), and the triangle moves illustrated in Figure 1 (c) 7]. An oriented divide link is an oriented link ambient isotopic to the link of some oriented divide.

The link of a divide is isotopic to the link of the oriented divide obtained from the divide by the doubling method, which is the first step of the visualization algorithm due to Hirasawa [8]. Let $P$ be a divide. For each branch $B$ of $P$, we draw the boundary of a 'very small' neighborhood of $B$ in the disk 




Figure 1: Inverse self-tangency moves and a triangle move

$D$, assigned with the clockwise orientation, as illustrated in Figure 2] where interrupted curves represent $\partial D$. In particular, we draw a 'sharp' around each double point of $P$, and draw a 'hairpin curve' around each point of $\partial P$. We suppose that such hairpin curves lie in the interior of $D$. We denote by $d(P)$ the oriented divide obtained by the above algorithm.



Figure 2: Doubling method

We review the second step of the visualization algorithm due to Hirasawa [8] We can apply his algorithm not only to divides but also to oriented divides. Let $Q$ be an oriented divide. A regular isotopy of $Q$ in the space of generic immersions does not change the ambient isotopy type of the link $L_{\text {ori }}(Q)$. Hence we may assume that oriented divides are linear with slope \pm 1 except near the 'corners', where a branch quickly changes its slope from \pm 1 to $\mp 1$. We draw a link diagram for $Q$ as below:

(1) We replace each double point of $Q$ with the crossing as illustrated at the top of Figure 3 .

(2) For each $x_{2}$-maximal (resp. $x_{2}$-minimal) point whose tangent vector has same orientation as the $x_{1}$-axis, we change the diagram as illustrated at the bottom of Figure 3. where interrupted curves represent $\partial D$, and 
horizontal arcs represent all arcs over (resp. under) the $x_{2}$-maximal (resp. $x_{2}$-minimal) point.

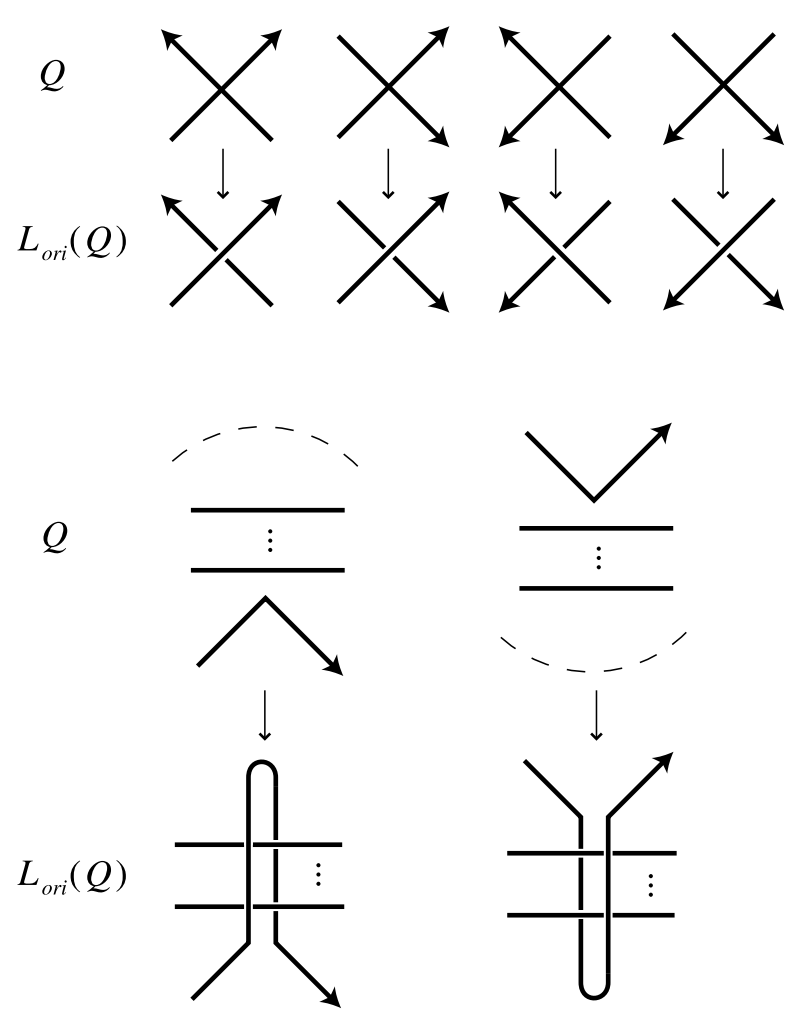

Figure 3: Local link diagrams obtained from oriented divides

In [8], Hirasawa showed that for any divide $P$, the diagram obtained by the above algorithm represents $L(P)$, the link of divide $P$. His argument implies that for any oriented divide $Q$, the diagram obtained by the second step of the above algorithm represents $L_{\text {ori }}(Q)$, the link of oriented divide $Q$. Therefore we obtain $L(P)=L_{\text {ori }}(d(P))$.

Remark 2.1 In 8], Hirasawa oriented the tangent bundle $T D$ by the coordinate $(x, u)=\left(x_{1}, x_{2}, u_{1}, u_{2}\right)$. Therefore the diagrams of links of divides in this paper are the mirror images of those in his paper, since the orientations of the 3-sphere are opposite. 


\section{$3 \quad$ Links of graph divides}

In this section, we construct graph divide links, that is, links associated with generic immersions of finite graphs in a 2-dimensional disk $D$, as an extension of the class of divide links.

A graph divide $P=(G, \varphi)$ is a generic immersion $\varphi: G \rightarrow D$ as follows or its image, where $G$ is a disjoint union of finite graphs and copies of the unit circle. Each graph may have loops and multiple edges. The singularities are only transversal double points of two arcs in edges and circles. We suppose that each point of $P \cap \partial D$ is the image of a vertex of degree 1 . We regard the unit interval as a finite graph. A branch of $P$ is the image of each component of $G$. We shall call the image of an interval component an interval branch, the image of a circle component a circle branch, and the image of a tree component a tree branch. The image of vertices of degree 1 might not lie in the boundary of the unit disk. We call such an image a free endpoint of $P$ and denote by $E_{P}$ the set of all free endpoints of $P$. We denote by $T_{P}$ the set of all vertices except free endpoints and points in $\partial D$. We denote $V_{P}=E_{P} \cup T_{P}$. If $T_{P}$ is empty and $\varphi$ is a non-relative immersion, $P$ is a free divide [7]. In [7, Gibson and Ishikawa considered free divides with only interval branches, but we consider both of interval and circle branches in this paper. We note that a divide is also a free divide [7, and that it is also a graph divide.

We extend the definition of links of divides as follows. In the case of free divides, the argument is almost same as the visualized definition for links of free divide due to Gibson and Ishikawa [7. We give 'signs' to vertices of a graph divide, because the link is not associated to a graph alone. Let $x$ be a vertex of $G$. We also denote the image $\varphi(x)$ by $x$. If $x$ lies in $\partial D, x$ does not need a sign. If $x$ is a point of $V_{P}$, we give $x$ a sign $\epsilon_{x}=+$ or $\epsilon_{x}=-$.

For a given graph divide $P=(G, \varphi)$ and given signs of vertices, we construct an oriented divide $d\left(P ;\left\{\epsilon_{x}\right\}_{x \in V_{P}}\right)$ by extending a doubling method as follows. For each branch $B$ of $P$, we draw the boundary of 'very small' neighborhood of $B$ in the disk $D$, assigned with the clockwise orientation, in the same way as that for divides, except near $x \in V_{P}$. Around $x \in E_{P}$ with $\epsilon_{x}=-$, we draw a 'hairpin curve', as illustrated in Figure 4 (b). Around $x \in E_{P}$ with $\epsilon_{x}=+$, we draw a 'loop', as illustrated in Figure 4 (a). Around $x \in T_{P}$ with $\epsilon_{x}=-$, we draw oriented curves such that each curve approaches $x$ along an edge and turns to its neighbor edge on the left, as illustrated in Figure 4 (d). Around $x \in T_{P}$ with $\epsilon_{x}=+$, we draw oriented curves such that each curve approaches $x$ along an edge and turns to its neighbor edge on the right, as illustrated in 
Figure 4(c). We denoted the obtained curves by $d\left(P ;\left\{\epsilon_{x}\right\}_{x \in V_{P}}\right)$ and call it the doubling of graph divide $P$ with signs $\left\{\epsilon_{x}\right\}_{x \in V_{P}}$.

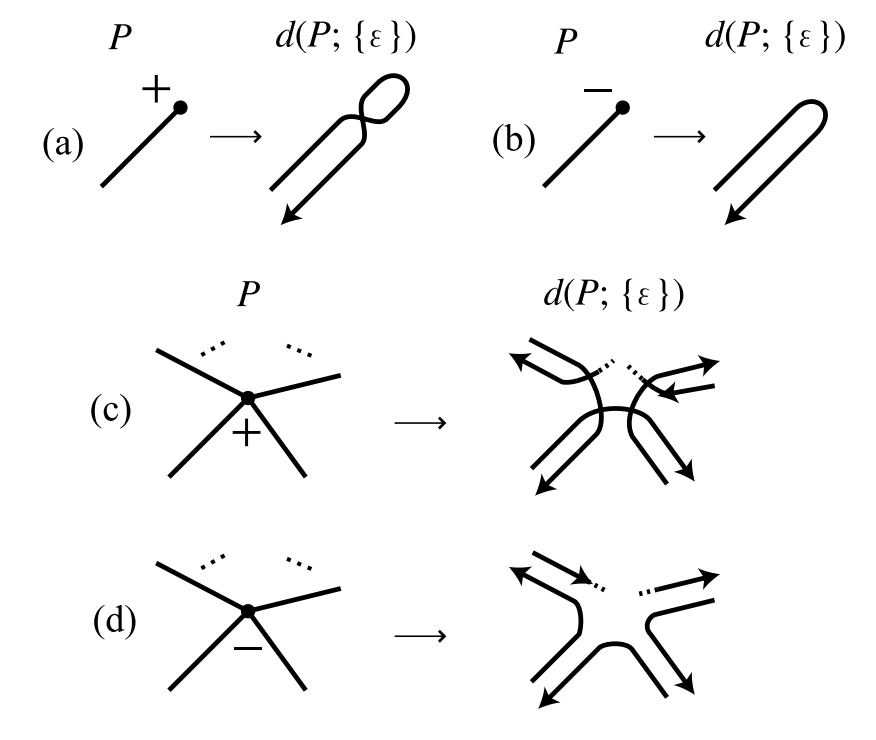

Figure 4: The doubling of graph divide around vertices

Definition 3.1 The link of a graph divide $P$ is the set given by

$$
L\left(P ;\left\{\epsilon_{x}\right\}_{x \in V_{P}}\right)=L_{\text {ori }}\left(d\left(P ;\left\{\epsilon_{x}\right\}_{x \in V_{P}}\right)\right) .
$$

We note that the link of a given graph divide depends on signs of vertices. For fixed signs of vertices $\left(\left\{\epsilon_{x}\right\}_{x \in V_{P}}\right)$, a regular isotopy of $P$ in the space of generic immersions does not change the isotopy type of the oriented divide $d\left(P ;\left\{\epsilon_{x}\right\}_{x \in V_{P}}\right)$, therefore it does not change the ambient isotopy type of the link $L\left(P ;\left\{\epsilon_{x}\right\}_{x \in V_{P}}\right)$. A graph divide link is the oriented link ambient isotopic to the link of some graph divide with some signs of vertices.

Furthermore some transformations on a graph divide do not change the isotopy type of the link.

Lemma 3.2 The transformations on a graph divide illustrated in Figure 5 do not change the ambient isotopy type of the link.

Proof For each transformation illustrated in Figure 5] we consider the doubling of the graph divides illustrated in Figure 4. They are changed to each other by diffeomorphisms of $D$, triangle moves and inverse self-tangency moves. 


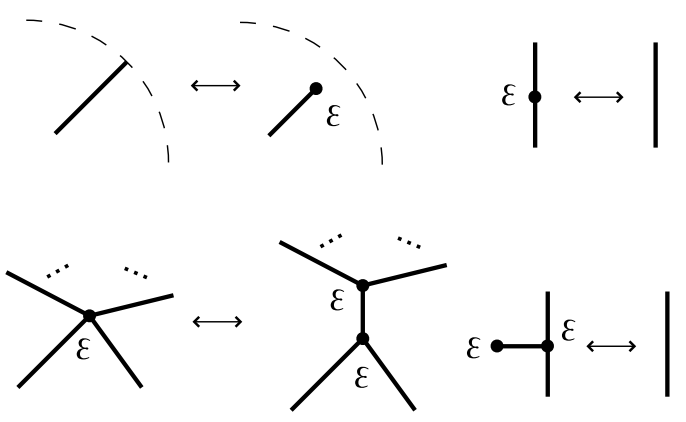

Figure 5: Transformations on a graph divide not changing the link type

By means of Lemma 3.2 for any graph divide $P=(G, \varphi)$, there exists a graph divide $P^{\prime}=\left(G^{\prime}, \varphi^{\prime}\right)$, where $G^{\prime}$ is an union of uni-trivalent graphs and copies of circles, such that $L\left(P^{\prime} ;\left\{\epsilon_{x}\right\}_{x \in V_{P^{\prime}}}\right)$ is ambient isotopic to $L\left(P ;\left\{\epsilon_{x}\right\}_{x \in V_{P}}\right)$.

The above definition of a graph divide is a natural extension of a (free) divide link. If $E_{P}=V_{P}$ holds, the link $L\left(P ;\left\{\epsilon_{x}\right\}_{x \in V_{P}}\right)$ is ambient isotopic to the link of a free divide defined by Gibson and Ishikawa [].

Example 3.3 (Cf. Gibson [5]) For a graph divide $P$ with signed vertices illustrated in Figure 6, the doubling of $P$ is illustrated as the right of $P$. Then the link of $P$ is the knot illustrated at the bottom of Figure [. It is known that this knot is not fibered if $n$ is a positive integer. Then it is not a divide link since divide links are all fibered.

Example 3.4 For a graph divide $P$ with signed vertices illustrated in Figure 7. the doubling of $P$ is illustrated as the right of $P$. Then the link of $P$ is the knot illustrated at the bottom of Figure 7 . This knot is the mirror image of $8_{21}$ in the table of Rolfsen [13. It is well known that the knot $8_{21}$ is fibered.

In the next section, we compare the above definition of the link of a graph divide with the construction of the link of a tree divide defined by Gibson in [5]. We use the following lemma, where we denote by $\bar{Q}$ the oriented divide obtained from a given oriented divide $Q$ by reversing the orientations of all branches. Gibson and Ishikawa showed as Proposition 3.1 in [6] that $L_{\text {ori }}(\bar{Q})$ is the same link as $L_{\text {ori }}(Q)$ but with the opposite orientations on all components of $L_{\text {ori }}(Q)$.

Lemma 3.5 Let $P=\left(G_{1} \sqcup G_{2}, \varphi\right)$ be a graph divide and we denote $\left(G_{j},\left.\varphi\right|_{G_{j}}\right)$ by $P_{j}=\left(G_{j}, \varphi_{j}\right)$ for $j=1,2$. We give each vertex $x \in V_{P}=V_{P_{1}} \cup V_{P_{2}}$ a sign 

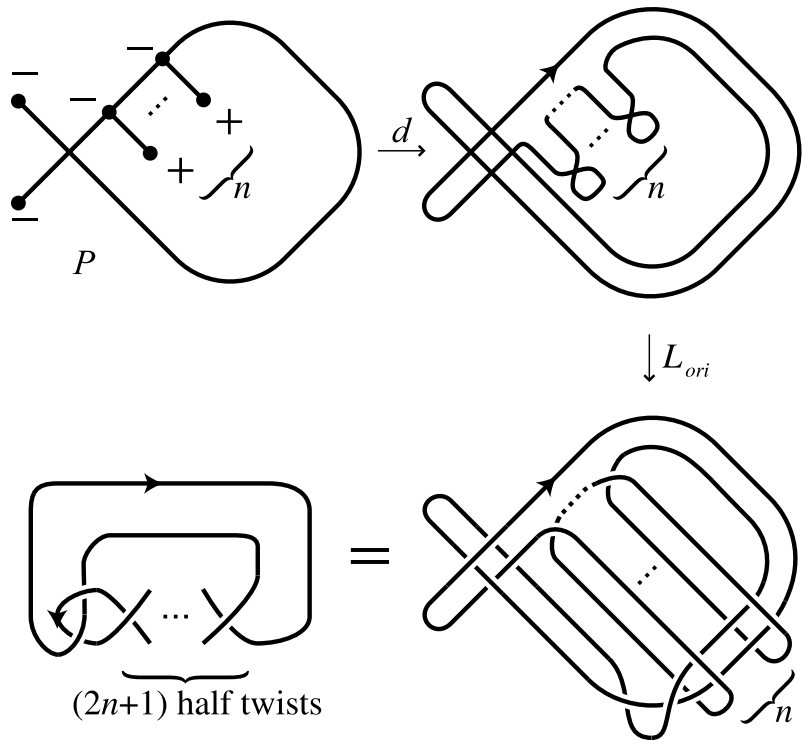

Figure 6: An example of a (non-fibered) graph divide knot
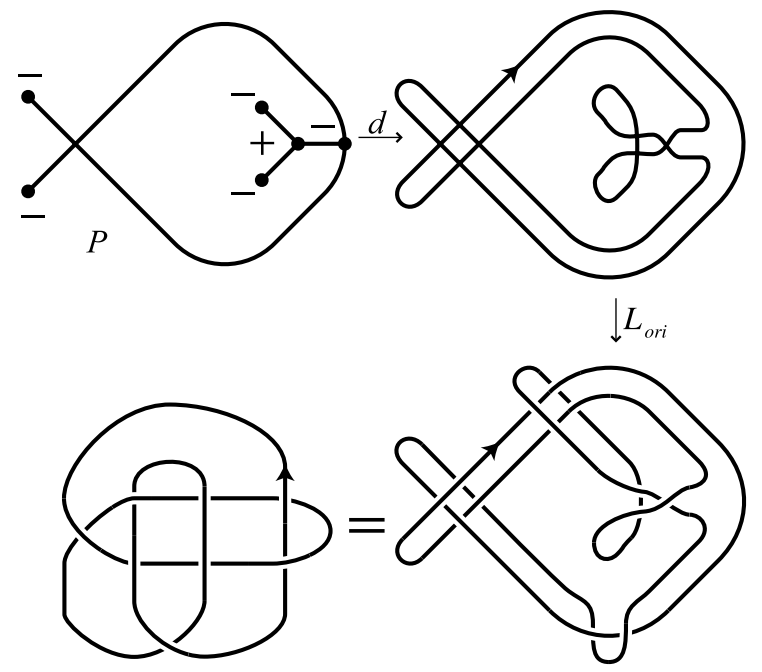

Figure 7: An example of a graph divide knot

$\epsilon_{x}$. Then the link $L\left(P ;\left\{\epsilon_{x}\right\}_{x \in V_{P_{1}}} \cup\left\{-\epsilon_{x}\right\}_{x \in V_{P_{2}}}\right)$ is ambient isotopic to the link $L_{\text {ori }}\left(d\left(P_{1} ;\left\{\epsilon_{x}\right\}_{x \in V_{P_{1}}}\right) \cup \overline{d\left(P_{2} ;\left\{\epsilon_{x}\right\}_{x \in V_{P_{2}}}\right)}\right)$, where $\left\{-\epsilon_{x}\right\}_{x \in V_{P_{2}}}$ is the set of the signs of $x \in V_{P_{2}}$ defined by $-\epsilon_{x}$. 
Proof By Lemma 3.2. we may suppose that $P \cap \partial D=\emptyset$ and the degree of each vertex of $G$ is 1 or 3 . We transform each part of $\overline{d\left(P_{2} ;\left\{\epsilon_{x}\right\}_{x \in V_{P_{2}}}\right)}$ as illustrated in Figure 8 (a), (b), (c), (d), and (f) by inverse self-tangency moves and triangle moves, and as illustrated in Figure 8 (e) and (g) by diffeomorphisms of $D$. We denote by $Q_{2}$ this new oriented divide obtained from $\overline{d\left(P_{2} ;\left\{\epsilon_{x}\right\}_{x \in V_{P_{2}}}\right)}$. Each of the parts in the small disks with dotted boundary in Figure 8 is same as the assigned part of the doubling of $d\left(P_{2} ;\left\{-\epsilon_{x}\right\}_{x \in V_{P_{2}}}\right)$. We remove the double points of $Q_{2}$ in the exterior of such disks by inverse selftangency moves as illustrated in Figure $8(\mathrm{~h})$. The finally obtained oriented divide is same as $d\left(P_{2} ;\left\{-\epsilon_{x}\right\}_{x \in V_{P_{2}}}\right)$. Then the oriented divide $d\left(P_{1} ;\left\{\epsilon_{x}\right\}_{x \in V_{P_{1}}}\right) \cup$ $\overline{d\left(P_{2} ;\left\{\epsilon_{x}\right\}_{x \in V_{P_{2}}}\right)}$ is changed to $d\left(P ;\left\{\epsilon_{x}\right\}_{x \in V_{P_{1}}} \cup\left\{-\epsilon_{x}\right\}_{x \in V_{P_{2}}}\right)$ by diffeomorphisms of $D$, inverse self-tangency moves, and triangle moves. Therefore the link $L_{\text {ori }}\left(d\left(P_{1} ;\left\{\epsilon_{x}\right\}_{x \in V_{P_{1}}}\right) \cup \overline{d\left(P_{2} ;\left\{\epsilon_{x}\right\}_{x \in V_{P_{2}}}\right)}\right)$ is ambient isotopic to $L\left(P ;\left\{\epsilon_{x}\right\}_{x \in V_{P_{1}}} \cup\right.$ $\left.\left\{-\epsilon_{x}\right\}_{x \in V_{P_{2}}}\right)$.

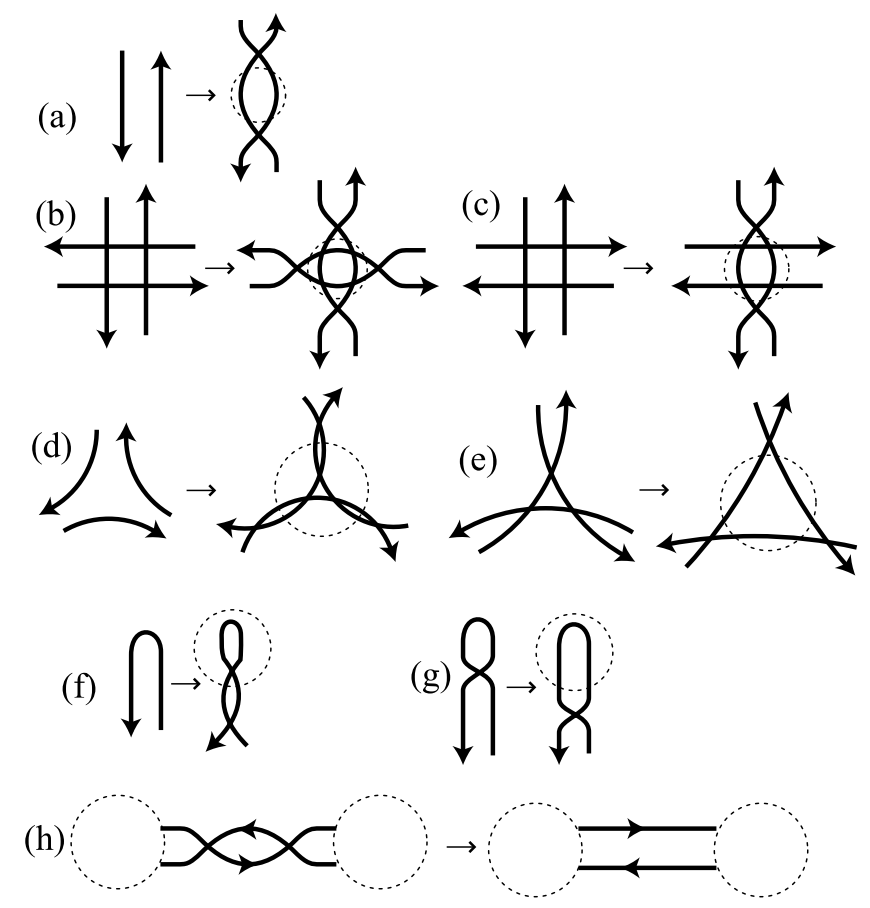

Figure 8: Transformations on $\overline{d\left(P_{2} ;\left\{\epsilon_{x}\right\}_{x \in V_{P_{2}}}\right)}$

Remark 3.6 Let $P=(I, \varphi)$ be a free divide with a single interval branch and $V_{P}=\left\{x_{1}, x_{2}\right\}$. In [7] Gibson and Ishikawa said that the sign of $P$ is even 
if $\epsilon_{x_{1}}=\epsilon_{x_{2}}$ holds, and otherwise odd. They showed that the isotopy class of the knot of such a free divide $P$ depends only on the sign of $P$. Actually, the orientation of $\left.L\left(P ;\left\{-\epsilon_{x_{1}},-\epsilon_{x_{2}}\right)\right\}\right)$ is the reverse of $\left.L\left(P ;\left\{\epsilon_{x_{1}}, \epsilon_{x_{2}}\right)\right\}\right)$. These facts are included in Lemma 3.5

\section{A relation with the construction due to Gibson}

We call a graph divide $P=(G, \varphi)$ a tree divide if $G$ is an union of trees [5]. In [5] Gibson defined the link of a tree divide by the different argument from that of the link of a graph divide in this paper. In this section, we review his definition and show that tree divide links defined by him are represented as graph divide links defined in Section 3.

For a given tree divide $P=(G, \varphi)$, Gibson defined a doubling of $P$ by the following argument. We assume that an union of trees $G$ may have isolated vertices. We construct a new non-oriented divide $\Delta(P)$ as follows. For each branch $B$ of $P$, we draw the boundary of 'very small' neighborhood of $B$ in the disk $D$ except near $x \in V_{P}$ as illustrated in Figure 9 (a), (b), and (c), in the almost same way as that for the doubling in Section 2. In this step we do not give any orientation. Around each vertex we connect these curves as illustrated in Figure 9 as follows. Around each isolated vertex, we draw a small circle as illustrated in Figure 9(d). Around $x \in E_{P}$, we draw a 'hairpin curve' as illustrated in Figure 9 (e). Around each vertex of degree 2, we draw two curves which cross each other once transversely as illustrated in Figure 9 (f). Around each of the remaining vertices, as illustrated in Figure 9 (g), we draw non-oriented curves the same way as the doubling around the vertex signed '- ' in the definition of a doubling of graph divide in Section 3

We give $\Delta(P)$ an arbitrary orientation $o$. Then we obtain an oriented divide $(\Delta(P), o)$. Gibson defined the link of a tree divide $P$ as the link $L_{\text {ori }}(\Delta(P), o)$.

Applying the above argument to an immersion of the graphs which are not trees is not a natural extension of the original definition of the link of a divide, because if we do that, the doubling of some regular arc of $P$ might induce two parallel arcs with same orientation after we orient $\Delta(P)$. By the following result, we see that our definition of links of graph divides is a natural extension of links of divides and tree divides.

Proposition 4.1 For any tree divide $P$, the link $L_{o r i}(\Delta(P), o)$ can be represented as a graph divide link defined in Section [3. 
(a)

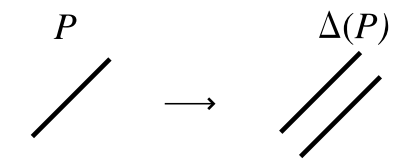

(b)

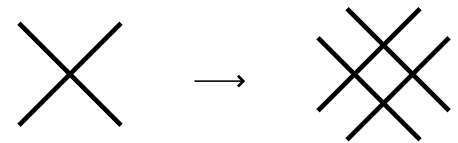

(c)

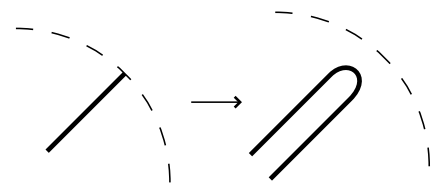

(d)

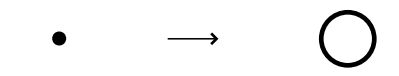

(e)

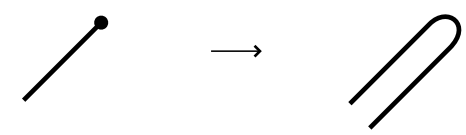

(f)

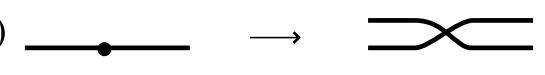

(g)
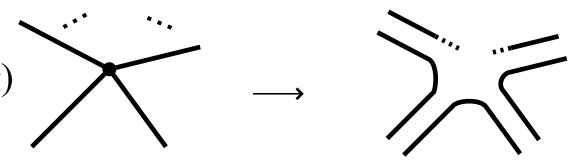

Figure 9: The doubling $\Delta(P)$ of a tree divide $P$

Proof Let $P=(G, \varphi)$ be a tree divide. By definition of the link of a tree divide, each isolated vertex may be replaced with a small embedded interval, and we may suppose that $P$ does not intersect $\partial D$ and the degree of each vertex of $G$ is 1,2 , or 3 . We construct a new tree divide $P_{0}$ and give the sign $\epsilon=+,-$ or the symbol $h$ to each vertex by replacing neighborhood of vertices as follows:

(1) We give the sign ' + ' to each vertex of degree 3 adjacent to three vertices of degree 2, and remove these vertices of degree 2, as illustrated in Figure 10 (a). We give the sign '- ' to each of the other vertices of degree 3 , as illustrated in Figure 10 (b).

(2) We give the sign + to each endpoint adjacent to a vertex of degree 2, and remove this vertex of degree 2, as illustrated in Figure 10 (c). We 


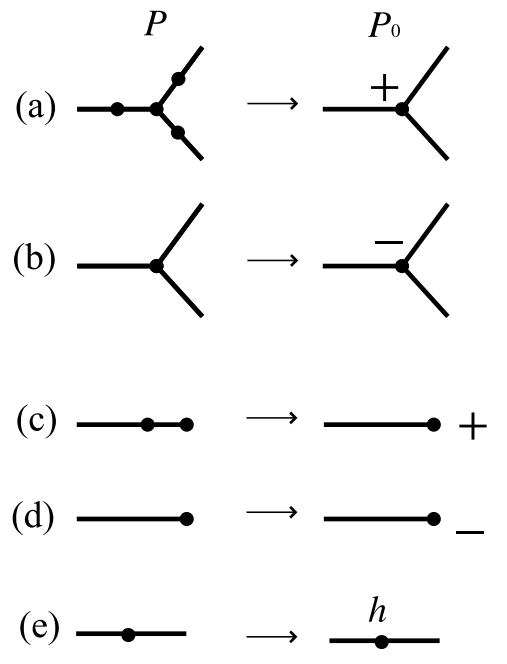

Figure 10: Transformations from $P$ to $P_{0}$

give the sign ' - ' to each of the other endpoints, as illustrated in Figure 10 (d).

(3) After applying the above two steps, we give the symbol ' $h$ ' to each of the other vertices of degree 2 , in order to distinguish the doubling around a vertex of degree 2 in this section from that defined in Section 3.

We define the doubling of $P_{0}, \Delta\left(P_{0} ;\left\{\eta_{x}\right\}_{x \in V_{P_{0}}}\right)$ as $\Delta(P)$, where $\eta_{x}$ is $h$ or + or - defined for each $x \in V_{P_{0}}$ as above. The moves (c) and (d) in Figure 11$]$ reduce the number of the vertices of degree 2 , changing the sign of the endpoint. The moves (a) and (b) in Figure 11] can bring the vertices of degree 2 close to the endpoints, changing the sign of the vertex of degree 3. By repeating these moves finitely many times, We obtain a tree divide $P_{1}=\left(G_{1}, \varphi\right)$ which has no vertex of degree 2 , and obtain signs of vertices $\left\{\epsilon_{x}\right\}_{x \in V_{P_{1}}}$.

Each of the transformations illustrated in Figure 11 does not change the doubling of tree divides up to inverse self-tangency moves and triangle moves. Then, for any orientation $o$ of $\Delta(P)$, the oriented divide $(\Delta(P), o)$ can be transformed to $\Delta\left(P_{1} ;\left\{\epsilon_{x}\right\}_{x \in V_{P_{1}}}\right)$ with some orientation $o_{1}$ by inverse self-tangency moves and triangle moves. The oriented divide $d\left(P_{1} ;\left\{\epsilon_{x}\right\}_{x \in V_{P_{1}}}\right)$ is also obtained as $\Delta\left(P_{1} ;\left\{\epsilon_{x}\right\}_{x \in V_{P_{1}}}\right)$ with some orientation $o_{1}^{\prime}$. For each branch $B$ of $P_{1}$ with $\left.o_{1}\right|_{\Delta\left(B ;\left\{\epsilon_{x}\right\}_{x \in V_{B}}\right)} \neq\left. o_{1}^{\prime}\right|_{\Delta\left(B ;\left\{\epsilon_{x}\right\}_{x \in V_{B}}\right)}$, we change the signs of all vertices of $B$. We denote new signs by $\left\{\epsilon_{x}^{\prime}\right\}_{x \in V_{P_{1}}}$. By Lemma 3.5 the link $L\left(P_{1} ;\left\{\epsilon_{x}^{\prime}\right\}_{x \in V_{P_{1}}}\right)$ is ambient isotopic to $L_{o r i}\left(\Delta\left(P_{1} ;\left\{\epsilon_{x}\right\}_{x \in V_{P_{1}}}\right), o_{1}\right)$. Therefore it is ambient isotopic to the link $L_{\text {ori }}(\Delta(P), o)$. 
(a)

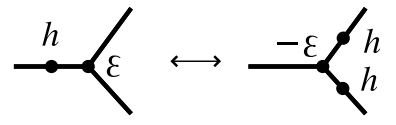

(b) $\stackrel{h}{ } \mid \leftrightarrow \frac{h}{}$

(c) $\stackrel{h}{\longrightarrow} \varepsilon \longrightarrow \longrightarrow$

(d) $\stackrel{h h}{\longrightarrow} \longrightarrow$

Figure 11: The algorithm to remove vertices of degree 2

\section{Quasipositivity}

By using the almost same argument as that due to Ishikawa [10, we have the following theorem.

Theorem 5.1 Links of graph divides are quasipositive.

In the proof of this theorem, we represent a graph divide as a tangle product constructed as follows. We restate a tangle product which Ishikawa defined for a divide in [10]. In this paper, we suppose that a tangle consists of some or no vertical lines and each of the following parts as illustrated in Figure 12.

(1) a pair of crossed curves illustrated in Figure 12 (a),

(2) a folding curve including an $x_{2}$-maximal point illustrated in Figure 12 (b),

(3) a folding curve including an $x_{2}$-minimal point illustrated in Figure 12 (c),

(4) a vertical line with a vertex of degree 1 illustrated in Figure 12 (d) and (e),

(5) a vertical line and a curve with a vertex of degree 3 illustrated in Figure 12 (f) and (g).

A tangle product is a product of these tangle with well-defined connections. The graph divide $P$ in Figure 16 is a tangle product representation for the graph divide of Example 3.4 illustrated in Figure 7. 


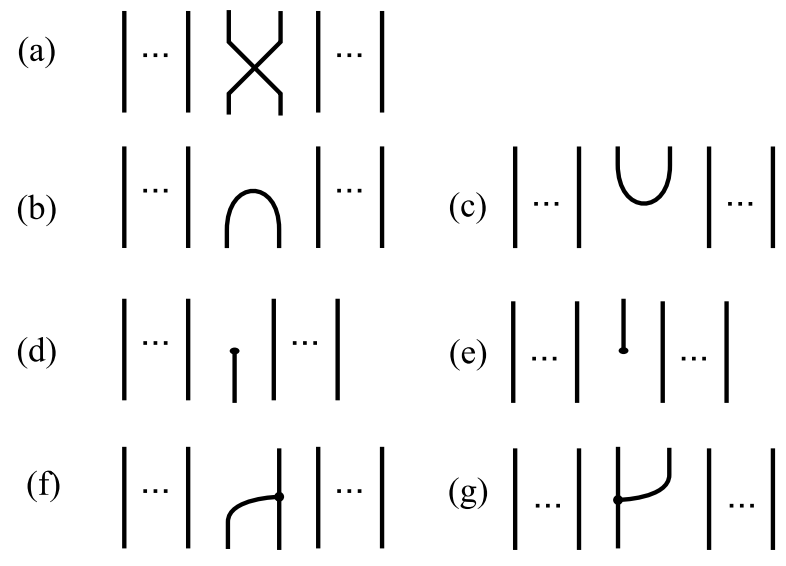

Figure 12: Tangles

Remark 5.2 In 10 Ishikawa constructed a tangle as horizontal lines instead of vertical lines. In his construction, some folding curves have marks in order to establish the Kauffman state model on divides. Here we do not mention about a mark since we do not need it in the present article.

Proof of Theorem [5.1 Let $P=(G, \varphi)$ be a graph divide. By Lemma 3.2. we may suppose that for each vertex of $G$, the degree is equal to 1 or 3 . A regular isotopy of $P$ in the space of generic immersions does not change the isotopy type of the link $L(P)$. Hence, we may assume that $P$ is represented as a tangle product. The horizontal arcs illustrated in Figure 13, 14, and 15] represent all parts over or under each of vertices, and $x_{2}$-maximal or minimal points. Furthermore, we may assume that for any $a \in[-1,1]$ the number of arc components of $\left(\left.p_{1}\right|_{P}\right)^{-1}(a)$ is less than 2 except when they are connected by the neighborhood of double points in $P$, where $p_{1}$ is the projection map from the disk $\mathrm{D}$ to the $x_{1}$-axis.

For a given graph divide $P$ and signs, we construct an immersed 2-manifold in the 3-sphere, $F\left(P ;\left\{\epsilon_{x}\right\}_{x \in V_{P}}\right)$, which consists of disks and bands as determined by the following algorithm.

(1) For each vertical arc $\alpha$ in $P$, we construct a wide band along $\alpha$ in the disk $D$ as illustrated in Figure 13 (a). This band is a part of 'disks' of $F\left(P ;\left\{\epsilon_{x}\right\}_{x \in V_{P}}\right)$, that is, each of such disks is the natural connected sum of the neighborhood of 1 -dimensional components of $\left(\left.p_{1}\right|_{P}\right)^{-1}(a)$ in $D$ for some $a$. 
(a)

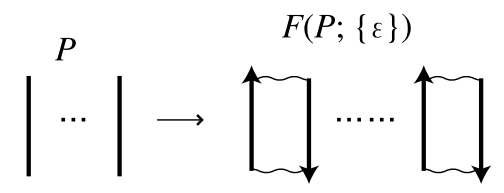

(b)

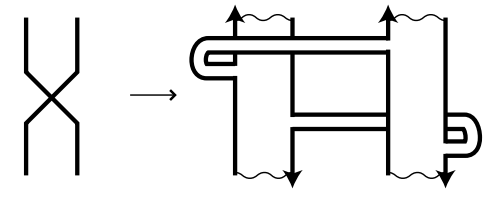

(c)

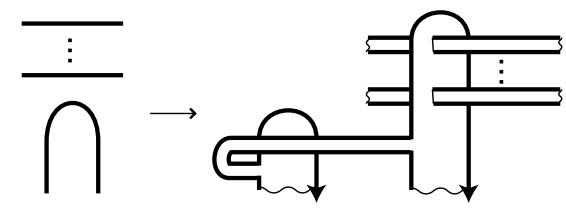

(d)

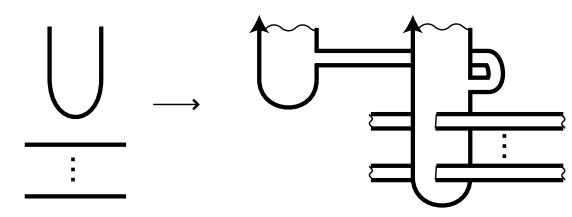

Figure 13: Steps 1, 2, and 3 of the construction of $F\left(P ;\left\{\epsilon_{x}\right\}_{x \in V_{P}}\right)$

(2) Around each double point of $P$, we construct two parts of disks and two narrow bands connecting them as illustrated in Figure 13 (b).

(3) Around each of $x_{2}$-maximal points and $x_{2}$-minimal points of $P$, we construct two parts of disks and a narrow band connecting them, as illustrated in Figure 13 (c) and (d). We construct intersections of a disk and the narrow bands corresponding to all small arcs in $P$ over the $x_{2}-$ maximal point or under the $x_{2}$-minimal point.

(4) Around each free endpoint of $P$, we construct a part of a disk and intersections of it and narrow bands corresponding to all small arcs in $P$ over or under the endpoint as illustrated in Figure 14.

(5) Around each vertex of degree 3 of $P$, we construct two parts of disks and a narrow band connecting them, as illustrated in Figure 15. We construct intersections of a disk and the narrow bands corresponding to all small arcs in $P$ over or under the vertex.

We note that the narrow bands constructed in Step 2, 3, and 5 may intersect some disks as explained in Step 3, 4, and 5. 


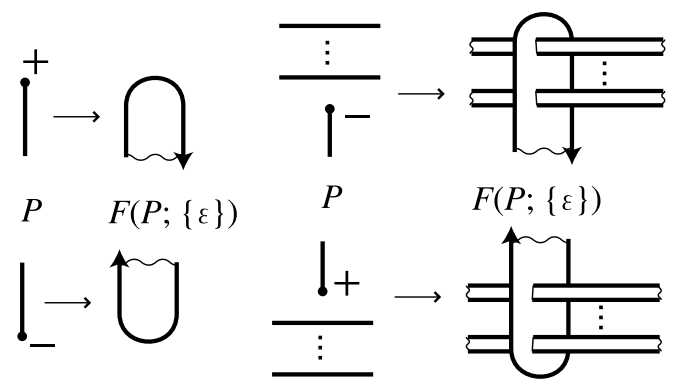

Figure 14: Step 4 of the construction of $F\left(P ;\left\{\epsilon_{x}\right\}_{x \in V_{P}}\right)$
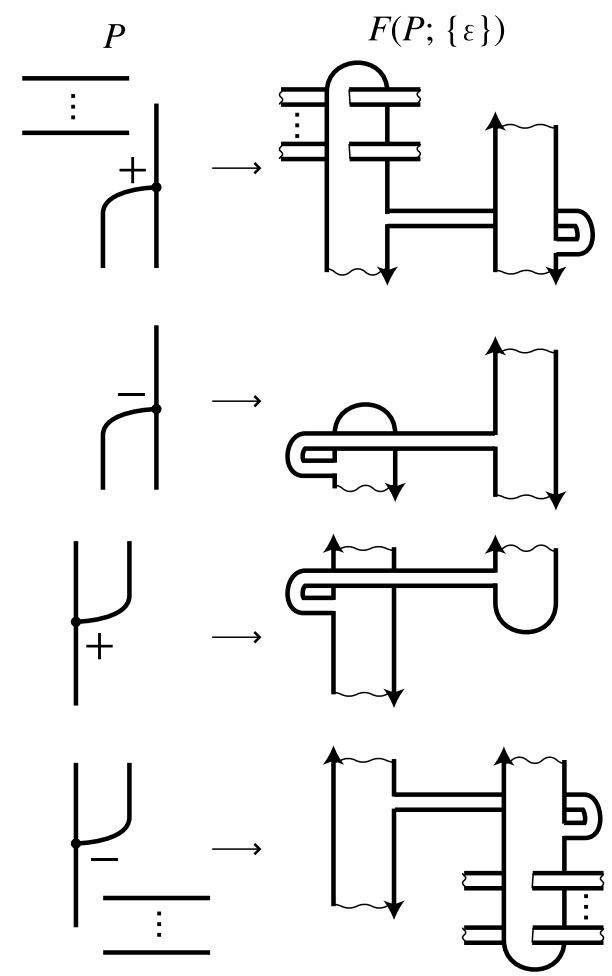

Figure 15: Step 5 of construction of $F\left(P ;\left\{\epsilon_{x}\right\}_{x \in V_{P}}\right)$

Figure [16 is an example of the pair of $P$ and $F\left(P ;\left\{\epsilon_{x}\right\}_{x \in V_{P}}\right)$. We suppose that the boundary of the above immersed 2-manifold, that is, $\partial F\left(P ;\left\{\epsilon_{x}\right\}_{x \in V_{P}}\right)$ is oriented clockwise in the diagram obtained by the above algorithm as illustrated in Figure 13, 14, and 15. We regard the diagram of $\partial F\left(P ;\left\{\epsilon_{x}\right\}_{x \in V_{P}}\right)$ as a closed 


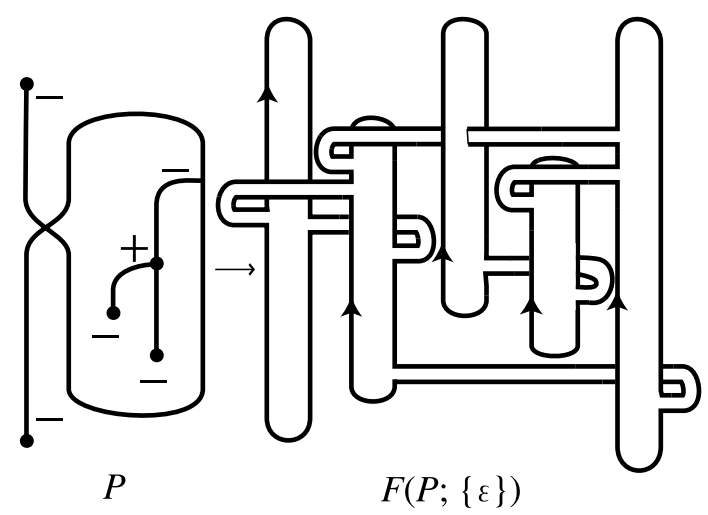

Figure 16: An example of $F\left(P ;\left\{\epsilon_{x}\right\}_{x \in V_{P}}\right)$

braid diagram. Each narrow band corresponds to a quasipositive band, a conjugate braid with a canonical generator of the braid group. Then $\partial F\left(P ;\left\{\epsilon_{x}\right\}_{x \in V_{P}}\right)$ is quasipositive. In the case of Figure 16, the link $\partial F\left(P ;\left\{\epsilon_{x}\right\}_{x \in V_{P}}\right)$ is the closure of the quasipositive braid

$$
\begin{aligned}
& \sigma_{1} \sigma_{4}\left(\sigma_{4}^{-1} \sigma_{3} \sigma_{2} \sigma_{3}^{-1} \sigma_{4}\right) \sigma_{1} \sigma_{3}\left(\sigma_{4}^{-1} \sigma_{3}^{-1} \sigma_{2} \sigma_{3} \sigma_{4}\right) \\
= & \sigma_{1} \sigma_{4}\left(\sigma_{2}^{-1} \sigma_{3} \sigma_{4} \sigma_{3}^{-1} \sigma_{2}\right) \sigma_{1} \sigma_{3}\left(\sigma_{2} \sigma_{3} \sigma_{4} \sigma_{3}^{-1} \sigma_{2}^{-1}\right),
\end{aligned}
$$

where $\sigma_{1}, \sigma_{2}, \sigma_{3}$, and $\sigma_{4}$ are the canonical generators of the 5-braid group. Comparing with the definition of the link of a graph divide, $\partial F\left(P ;\left\{\epsilon_{x}\right\}_{x \in V_{P}}\right)$ is ambient isotopic to $L\left(P ;\left\{\epsilon_{x}\right\}_{x \in V_{P}}\right)$. Therefore the link $L\left(P ;\left\{\epsilon_{x}\right\}_{x \in V_{P}}\right)$ is quasipositive.

The braid index of a link $L$ is the minimal number of strings required to represent $L$ as a closed braid. The above argument implies the following result.

Proposition 5.3 Let $P$ is a graph divide represented as a tangle product in the proof of Theorem 5.1. Let $v$ be the number of vertices of $P$ and $m$ be the number of $x_{2}$-maximal points and $x_{2}$-minimal points of $P$. Then the braid index of the link of $P$ is not greater than $\frac{1}{2}(v+2 m)$.

Remark 5.4 In private communication, Ishikawa found that any closed positive braid can be represented as a graph divide link. For a given closed positive braid $L$, we draw a uni-trivalent graph which is homotopic to the canonical Seifert surface of $L$, and for endpoints of each edge corresponding to a positive 

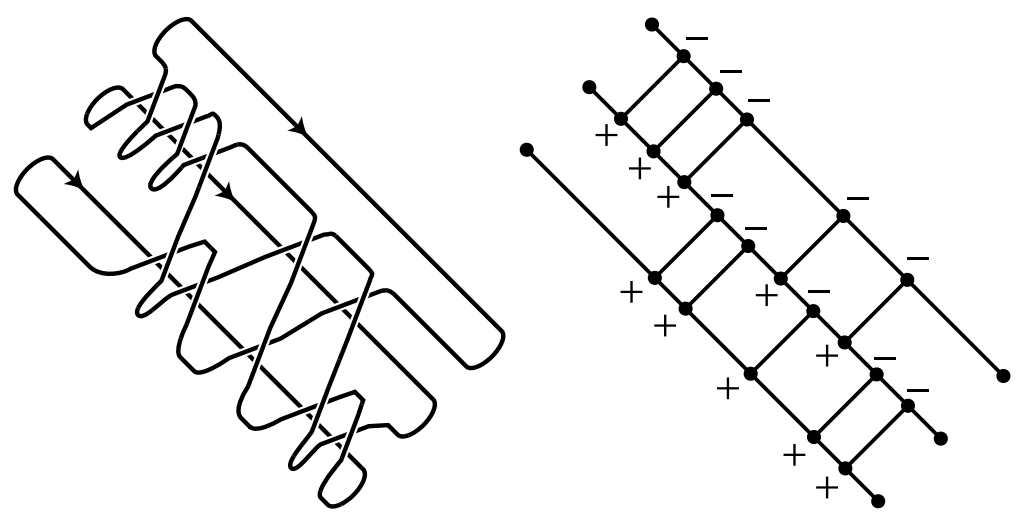

Figure 17: A closed positive braid and a graph divide representing it

crossing we give the different signs. For example, the knot $10_{139}$ in the table of Rolfsen [13] is the closure of the positive braid $\sigma_{1}{ }^{2} \sigma_{2} \sigma_{1} \sigma_{2} \sigma_{1}{ }^{2} \sigma_{2}{ }^{3}$, where $\sigma_{1}$ and $\sigma_{2}$ are the canonical generators of the 3 -braid group. By this word, we draw a graph and give signs of vertices as illustrated in Figure 17. The link of this graph divide is the knot $10_{139}$. Actually, the knot $10_{139}$ is the knot of each divide in Figure 18, 3, 7].

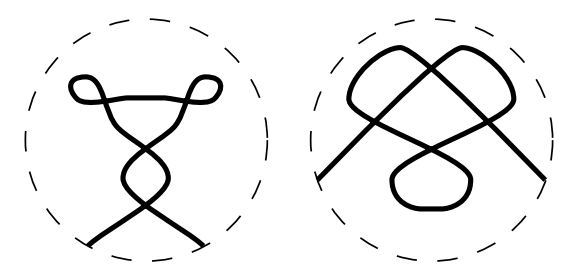

Figure 18: Divides which represent the knot $10_{139}$

\section{Four-dimensional invariants}

In this section, we determine some link invariants for graph divide links.

Let $L$ be an oriented link, and let $F \subset B^{4}$ be a smooth, oriented 2-manifold with $\partial F=L$, where $B^{4}$ is the 4 -ball bounded by $S^{3}$. We suppose that $F$ has no closed components, but $F$ is not assumed to be connected. We denote by $\chi_{s}(L)$ the greatest value of the euler characteristic $\chi(F)$ for such 2-manifolds $F \subset B^{4}$, and we call this invariant the slice euler characteristic. In [16], Rudolph showed the following equality. 
Theorem 6.1 [16] Let $L$ be the closure of a quasipositive braid with $n$ strings and $k$ quasipositive bands. Then we have $\chi_{s}(L)=n-k$.

Combining Theorem 6.1 and the proof of Theorem [5.1, we obtain the following result.

Proposition 6.2 Let $P=(G, \varphi)$ be a graph divide. Let $\delta(P)$ be the number of double points of $P$. Then the slice euler characteristic of the link of $P$ is $\chi(G)-2 \delta(P)$.

Proof Let $P=(G, \varphi)$ be a graph divide and $\delta$ the number of double points of $P$. We may assume that $F\left(P ;\left\{\epsilon_{x}\right\}_{x \in V_{P}}\right)$ in the proof of Theorem 5.1 is the projection image of a $2-$ manifold in $B^{4}-\{*\}$ to $S^{3}$. We also denote this 2-manifold by $F\left(P ;\left\{\epsilon_{x}\right\}_{x \in V_{P}}\right)$. By Theorem 6.1 and the construction of $F\left(P ;\left\{\epsilon_{x}\right\}_{x \in V_{P}}\right)$ we have

$$
\chi_{s}\left(L\left(P ;\left\{\epsilon_{x}\right\}_{x \in V_{P}}\right)\right)=\chi\left(F\left(P ;\left\{\epsilon_{x}\right\}_{x \in V_{P}}\right)\right) .
$$

By means of constructions of an immersed 2-manifold $F\left(P ;\left\{\epsilon_{x}\right\}_{x \in V_{P}}\right)$, we have

$$
\chi\left(F\left(P ;\left\{\epsilon_{x}\right\}_{x \in V_{P}}\right)\right)=\chi(G)-2 \delta(P) .
$$

Remark 6.3 The slice euler characteristic of the link of graph divide does not depend on signs of vertices by the above proposition.

Let $L$ be an $r$-component link. The four-dimensional clasp number of $L$ is the minimum number of the double points for transversely immersed $r$ disks in $B^{4}$ with boundary $L$ and with only finite double points as singularities [11. The author showed in [1] that the inequality $c_{s}(L) \geq\left(r-\chi_{s}(L)\right) / 2$ holds for any link $L$. In particular, if $G$ is a disjoint union of intervals and trees, the 4-dimensional clasp number of the link of the graph divide $P=(G, \varphi)$ is determined as below.

Corollary 6.4 Let $P=(G, \varphi)$ be a graph divide. Let $\delta(P)$ be the number of double points of $P$. If $G$ is a disjoint union of intervals and trees, then the four-dimensional clasp number of the links of $P$ is $\delta(P)$. 


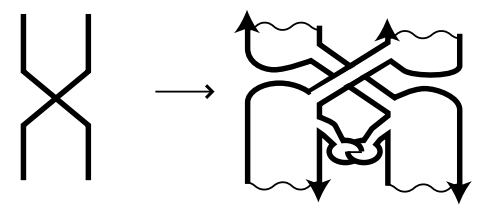

Figure 19: Construction of immersed disks

Proof By the above, the 4-dimensional clasp number of the links of $P$ is not less than $\delta(P)$. We show that the 4-dimensional clasp number of the links of $P$ is not greater than $\delta(P)$.

For a given tree divide $P$ and signs of vertices $\left\{\epsilon_{x}\right\}_{x \in V_{P}}$, we construct immersed disks in the 3-sphere by the almost same argument as that for the 2-manifold $F\left(P ;\left\{\epsilon_{x}\right\}_{x \in V_{P}}\right)$ in Section 5] For $P$ except near double points, we construct same parts of $F\left(P ;\left\{\epsilon_{x}\right\}_{x \in V_{P}}\right)$. Around each double point of $P$, we construct parts of disks with a clasp as illustrated in Figure 19. Non-vertical narrow bands may intersect some disks as explained in Step 3, 4, and 5 of the construction of $F\left(P ;\left\{\epsilon_{x}\right\}_{x \in V_{P}}\right)$. As an example the immersed disk in Figure 20 is obtained from the pair of $P$ and $F\left(P ;\left\{\epsilon_{x}\right\}_{x \in V_{P}}\right)$ in Figure 16] by the above algorithm. We regard the obtained diagram of immersed disks as a diagram of disks immersed in $B^{4}$ with boundary $L\left(P ;\left\{\epsilon_{x}\right\}_{x \in V_{P}}\right)$. Then we have $c_{s}\left(L\left(P ;\left\{\epsilon_{x}\right\}_{x \in V_{P}}\right)\right) \leq$ $\delta(P)$.

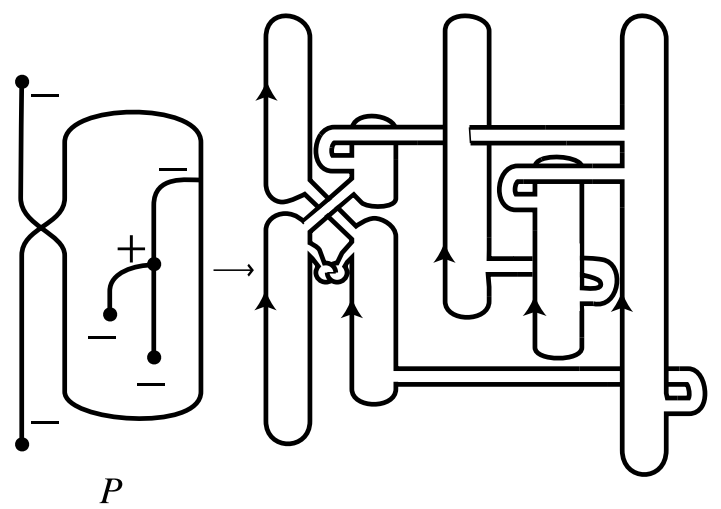

Figure 20: An example of the immersed disk

Remark 6.5 In Example 3.4 we represent the knot $8_{21}$ as a graph divide knot. It is known that the genus of this knot is 2. In [17] Shibuya showed that 
for any link, the 3-dimensional clasp number is not less than the genus. Then the 3-dimensional clasp number of $8_{21}$ is not less than 2 , and actually equal to 2. By Corollary 6.4 we have $c_{s}\left(8_{21}\right)=1$. Therefore Corollary 6.4 cannot determine the 3 -dimensional clasp numbers of graph divide links generally.

Remark 6.6 A'Campo [2], Gibson and Ishikawa [7] showed that if $P$ is a divide or a free divide without circle branches, the unknotting number of the link of $P$ is also equal to the number of double points of $P$. In [7], Gibson and Ishikawa introduced a good unknotting operation for $P$. Furthermore in [5] Gibson showed that the unknotting number of the link of a tree divide with some conditions is equal to the number of double points.

In [7, Gibson and Ishikawa checked that there exist free divide links with no divide representation and that the knot of the free divide with only one double point must be the trefoil or the mirror image of the knot $5_{2}$ in the table due to Rolfsen 13. Then Corollary 6.4 implies that each of the knots of Example 3.3 for $n \geq 0$ and Example 3.4 cannot be represented by any free divide (cf. [5]). Therefore the class of graph divide links is a real extension of the class of free divide links.

Then we are interested in the problem of the existence of the quasipositive link with no graph divide representation. By Proposition [6.2, we obtain the following result.

Theorem 6.7 If a graph divide knot $K$ is slice, then $K$ is trivial. Therefore, there exists a quasipositive link which is not a graph divide link.

In the proof of the above theorem, we use the following lemma.

Lemma 6.8 If a graph $G$ is a connected tree and the map $\varphi: G \rightarrow D$ is an embedding, then the link of $P=(G, \varphi)$ is a trivial knot.

Proof By moves illustrated in Figure 5. $G$ can be transformed to an arc $A$ embedded in $D$. By Lemma 3.2 the link of $P=(G, \varphi)$ is ambient isotopic to the link of $A$.

Proof of Theorem 6.7 We suppose that $K=L\left((G, \varphi) ;\left\{\epsilon_{x}\right\}_{x \in V_{P}}\right)$ is a slice knot. The graph $G$ is connected since $K$ is a knot. Hence the euler characteristic of $G$ is not greater than 1. By Proposition 6.2 the slice euler characteristic of $K$ is $\chi(G)-2 \delta$ where $\delta$ is the number of double points of $P$. We have 
$\chi(G)=2 \delta+1$ since the knot $K$ is slice. Then the euler characteristic of $G$ must be 1 and $\delta$ must be 0 . Therefore $G$ is a connected tree and $\varphi$ is an embedding. By Lemma 6.8 is a trivial knot.

The mirror image of the knot $8_{20}$ in the table due to Rolfsen 13 is the closure of the quasipositive braid $\sigma_{1}^{3} \sigma_{2} \sigma_{1}^{-3} \sigma_{2}$, where $\sigma_{1}$ and $\sigma_{2}$ are canonical generators of the 3-braid group. This knot is non-trivial and slice. Then it is an example of a quasipositive link which is not a graph divide link.

Remark 6.9 A strongly quasipositive braid is the product of positive bands. A positive band is a braid $\sigma_{i j}=\sigma_{i} \cdots \sigma_{j-1} \sigma_{j} \sigma_{j-1}{ }^{-1} \cdots \sigma_{i}^{-1}$, where $\sigma_{k}$ 's are canonical generators of the braid group and $i$ is less than $j$. A strongly quasipositive link is the closure of a strongly quasipositive braid. For any strongly quasipositive link, the slice euler characteristic is equal to the euler characteristic. Hirasawa recently showed that any divide link is strongly quasipositive [9]. The knot of Example 3.4 is fibered, but not strongly quasipositive, because the slice euler characteristic is -1 but the euler characteristic is -3 , so the slice euler characteristic is not equal to the euler characteristic.

\section{References}

[1] N A'Campo, Real deformations and complex topology of plane curve singularities, Annales de la Faculté des Sciences de Toulouse 8 (1999) 5-23 MathReview

[2] N A'Campo, Generic immersions of curves, knots, monodromy and gordian number, Publ. Math. IHES 88 (1998) 151-169 MathReview

[3] N A'Campo, Planer trees, slalom curves and hyperbolic knots, Publ. Math. IHES 88 (1998) 171-180 MathReview

[4] M Boileau, S Orevkov, Quasipositivité d'une courbe analytique dans une boule pseudo-convexe, C. R. Acad. Sci. Paris 332 (2001) 825-830 MathReview

[5] W Gibson, Links and Gordian numbers associated with generic immersions of trees, Proceedings of Art of Low Dimensional Topology VII (2001) 27-35

[6] W Gibson, M Ishikawa, Links of oriented divides and fibrations in link exteriors, Osaka J. Math. 39 (2002) 681-703 MathReview

[7] W Gibson, M Ishikawa, Links and Gordian numbers associated with generic immersions of intervals, Topol. Appl. 123 (2002) 609-636 MathReview

[8] M Hirasawa, Visualization of A'Campo's fibered links and unknotting operations, Topol. Appl. 121 (2002) 287-304 MathReview

[9] M Hirasawa, in preparation.

[10] M Ishikawa, The $\mathbb{Z}_{2}$-coefficient Kauffman state model on divides, preprint 
[11] T Kawamura, On unknotting numbers and four-dimensional clasp numbers of links, Proc. Amer. Math. Soc. 130 (2002) 243-252 MathReview

[12] T Kawamura, Quasipositivity of links of divides and free divides, Topol. Appl. 125 (2002) 111-123 MathReview

[13] D Rolfsen, Knots and links, Mathematics Lecture Series 7, Publish or Perish, Berkeley, Calif. (1976) MathReview

[14] L Rudolph, Algebraic functions and closed braids, Topology 22 (1983) 191-201 MathReview

[15] L Rudolph, Totally tangential links of intersection of complex plane curves with round spheres, from: "Topology '90 (Columbus OH (1990)", de Gruyter, Berlin (1992) 343-349 MathReview

[16] L Rudolph, Quasipositivity as an obstruction to sliceness, Bull. Amer. Math. Soc. 29 (1993) 51-59 MathReview

[17] T Shibuya, Some relations among various numerical invariants for links, Osaka J. Math. 11 (1974) 313-322 MathReview

Department of Physics and Mathematics, Aoyama Gakuin University

5-10-1, Fuchinobe Sagamihara, Kanagawa 229-8558, Japan

Email: tomomi@gem.aoyama.ac.jp

Received: 2 January 2003 Revised: 1 June 2004 